\title{
STURM-LIOUVILLE ABSTRACT PROBLEMS FOR THE SECOND ORDER DIFFERENTIAL EQUATIONS IN A NON COMMUTATIVE CASE
}

\author{
M. Kaid ${ }^{1}$, K. Ould Melha ${ }^{1}$
}

${ }^{1}$ Université Abdelhamid Ibn Badis, Mostaganem, Algérie

E-mail: mohammed.kaid@univ-mosta.dz, ould_melha_khel@yahoo.fr

\begin{abstract}
In this paper we prove some new results on Sturm-Liouville abstract problems of the second order differential equations of elliptic type in a new non-commutative framework. We study the case when the second member belongs to a Sobolov space. Existence, uniqueness and optimal regularity of the strict solution are proved. This paper is naturally the continuation of the ones studied by Cheggag et al in the commutative case. We also give an example to which our theory applies.

Keywords: second-order abstract elliptic differential equations; Sturm-Liouville boundary conditions in non commutative cases; analytic semigroup; maximal regularity.
\end{abstract}

\section{Introduction}

In a complex Banach space $X$ consider the following second order differential coefficient-operator equation

$$
u^{\prime \prime}(x)+A u(x)-\omega u(x)=f(x), \quad x \in(0,1),
$$

together with the abstract Robin boundary conditions

$$
u^{\prime}(0)-H u(0)=d_{0}, u(1)=u_{1} .
$$

Here $A$ and $H$ are closed linear operators with domains $D(A)$ and $D(H)$ in $X, f$ belongs to $L^{p}(0,1 ; X)$ with $1<p<\infty, d_{0}$ and $u_{1}$ are given elements of $X$ and $\omega$ is some large positive number.

Our goal is to seek for a classical solution to (1) - (2), that is a function $u$ such that

$$
\left\{\begin{array}{l}
\text { i) } u \in W^{2, p}(0,1 ; X) \cap L^{p}(0,1 ; D(A)), \\
\text { ii) } u(0) \in D(H), \\
\text { iii) } u \text { satisfies }(1),(2) .
\end{array}\right.
$$

Consider some fixed $\omega_{0} \geqslant 0$ and for $\omega>\omega_{0}$ set

$$
A_{\omega}=A-\omega I \text {. }
$$

This paper is a natural continuation of [1] and [2], where the authors have studied (1) - (2) in a commutative framework, when

$$
\left.f \in L^{p}(0,1 ; X) \text { with } 1<p<\infty \text { and } f \in C^{\theta}([0,1] ; X) \text { with } \theta \in\right] 0,1[.
$$

In [1] authors have assumed that

$X$ is a $U M D$ space, 


$$
\left\{\begin{array}{l}
A_{\omega_{0}} \text { is a linear closed operator in } X,\left[0,+\infty\left[\subset \rho\left(A_{\omega_{0}}\right)\right. \text { and }\right. \\
\sup _{\lambda \geqslant 0}\left\|\lambda\left(A_{\omega_{0}}-\lambda I\right)^{-1}\right\|_{\mathcal{L}(X)}<+\infty
\end{array}\right.
$$

(note that the previous assumption implies that, for all $\omega \geqslant \omega_{0}$, the operator $Q_{\omega}=$ $-\left(-A_{\omega}\right)^{1 / 2}$ is an infinitesimal generator of a bounded analytic semigroup on $\left.X\right)$.

$$
\left\{\begin{array}{l}
\text { for any } \left.s \in \mathbb{R}, \quad\left(-A_{\omega_{0}}\right)^{i s} \in \mathcal{L}(X) \text { and there exists } \theta_{A} \in\right] 0, \pi[\text { such that } \\
\sup _{s \in \mathbb{R}}\left\|e^{-\theta_{A}|s|}\left(-A_{\omega_{0}}\right)^{i s}\right\|_{\mathcal{L}(X)}<+\infty
\end{array}\right.
$$

$$
\begin{aligned}
& \left\{\begin{array}{l}
H \text { is a linear closed operator in } X, \mathbb{R}_{-} \subset \rho(H) \text { and } \\
\sup _{\xi \geqslant 0}\left\|\xi(H+\xi I)^{-1}\right\|_{\mathcal{L}(X)}<+\infty,
\end{array}\right. \\
& \left(A_{\omega_{0}}-\lambda I\right)^{-1}(H+\xi I)^{-1}=(H+\xi I)^{-1}\left(A_{\omega_{0}}-\lambda I\right)^{-1}, \quad \lambda \geqslant 0, \xi \geqslant 0, \\
& \left\{\begin{array}{l}
\left.\exists C \geqslant 1, \exists \theta_{H} \in\right] 0, \pi\left[: \forall s \in \mathbb{R}, \quad(H)^{i s} \in \mathcal{L}(X)\right. \text { and } \\
\left\|H^{i s}\right\|_{\mathcal{L}(X)} \leqslant C e^{\theta_{H}|s|}
\end{array}\right. \\
& \left.\frac{\theta_{A}}{2}+\theta_{H} \in\right] 0, \pi[
\end{aligned}
$$

Assumptions (3) - (9) imply that

$$
Q_{\omega}-H \text { is closed and boundedly invertible, }
$$

and there exists $\omega_{*} \geqslant \omega_{0}$ such that, for all $\omega \geqslant \omega_{*}$, the operator $\Lambda_{\omega}$ defined by

$$
\left\{\begin{array}{l}
D\left(\Lambda_{\omega}\right)=D\left(Q_{\omega}\right) \cap D(H) \\
\Lambda_{\omega}=\left(Q_{\omega}-H\right)+e^{2 Q_{\omega}}\left(Q_{\omega}+H\right)
\end{array}\right.
$$

is also closed and boundedly invertible, see for instance [1, Proposition 7 and Lemma 8, pp. 987-988].

Under these hypotheses, the authors built the representation formula of the solution of (1), (2) in the form

$$
\begin{aligned}
& u(x)=\left(e^{x Q_{\omega}}-e^{(2-x) Q_{\omega}}\right) \Lambda_{\omega}^{-1} d_{0}+e^{(1-x) Q_{\omega}} u_{1}+\left(e^{x Q_{\omega}}-e^{(2-x) Q_{\omega}}\right)\left(Q_{\omega}+H\right) \Lambda_{\omega}^{-1} e^{Q_{\omega}} u_{1}+ \\
&+\frac{1}{2}\left(e^{x Q_{\omega}}-e^{(2-x) Q_{\omega}}\right)\left(Q_{\omega}+H\right) \Lambda_{\omega}^{-1} Q_{\omega}^{-1} \int_{0}^{1}\left(e^{s Q_{\omega}}-e^{(2-s) Q_{\omega}}\right) f(s) d s- \\
&-\frac{1}{2} e^{(1-x) Q_{\omega}} Q_{\omega}^{-1} \int_{0}^{1} e^{(1-s) Q_{\omega}} f(s) d s+\frac{1}{2} Q_{\omega}^{-1} \int_{0}^{x} e^{(x-s) Q_{\omega}} f(s) d s+\frac{1}{2} Q_{\omega}^{-1} \int_{x}^{1} e^{(s-x) Q_{\omega}} f(s) d s,
\end{aligned}
$$

and have proved the following result.

Theorem 1. Assume (3) - (9). Let $f \in L^{p}(0,1 ; X)$ with $1<p<\infty$. Then there exists $\omega_{*} \geqslant \omega_{0}$ such that for all $\omega \geqslant \omega_{*}$ the two following assertions are equivalent:

1. $\Lambda_{\omega}^{-1} d_{0}, u_{1} \in(D(A), X)_{\frac{1}{2 p}, p}$.

2. Problem (1), (2) has a strict solution u, that is,

$$
u \in W^{2, p}(0,1 ; X) \cap L^{p}(0,1 ; D(A))
$$

$u(0) \in D(H)$ and $u$ satisfies (1), (2). 
Moreover, in this case, $u$ is uniquely determined by (10).

Recently, in [3], the authors have developed an interesting new approach concerning some general Sturm-Liouville problems with the same Robin boundary condition in 0 . They have assumed that:

- $\Lambda_{\omega}$ is boundedly invertible.

- $\Lambda_{\omega}^{-1}$ is a regularizing operator in the sense that

$$
\Lambda_{\omega}^{-1}\left(D\left(Q_{\omega}\right)\right) \subset D\left(Q_{\omega}^{2}\right) .
$$

\section{New Considerations and Main Result}

Consider problem (1), (2). In this work we will suppose that

$X$ is a $U M D$ space,

$$
\begin{gathered}
\left\{\begin{array}{l}
A_{\omega_{0}} \text { is a linear closed operator in } X,\left[0,+\infty\left[\subset \rho\left(A_{\omega_{0}}\right)\right. \text { and }\right. \\
\sup _{\lambda \geqslant 0}\left\|\lambda\left(A_{\omega_{0}}-\lambda I\right)^{-1}\right\|_{\mathcal{L}(X)}<+\infty,
\end{array}\right. \\
\left\{\begin{array}{l}
\text { for any } \left.s \in \mathbb{R}, \quad\left(-A_{\omega_{0}}\right)^{i s} \in \mathcal{L}(X) \text { and there exists } \theta_{A} \in\right] 0, \pi[\text { such that } \\
\sup _{s \in \mathbb{R}}\left\|e^{-\theta_{A}|s|}\left(-A_{\omega_{0}}\right)^{i s}\right\|_{\mathcal{L}(X)}<+\infty,
\end{array}\right.
\end{gathered}
$$

and

$$
\left\{\begin{array}{l}
\exists \nu \in] 0, \frac{1}{2}\left[, \exists C>0: \forall \mu>0, \forall \omega \geqslant \omega_{0}, D\left(A_{\omega}\right) \subset D(H)\right. \\
\text { and }\left\|H\left(A_{\omega}-\mu I\right)^{-1}\right\|_{\mathcal{L}(X)} \leqslant \frac{C}{|\omega+\mu|^{1 / 2+\nu}} .
\end{array}\right.
$$

Observe that we have assumed that operator $Q$ is principal but we do not suppose the commutativity between $H$ and the resolvent of $A$.

This article is organized as follows. In Section 2 we do some consequences and present preliminary technical results. In Section 3 we recall the representation formula of the solution $u$ of $(1)-(2)$. In Section 4 we prove our main result. Finally, in Section 5 we give some examples of application to boundary value problems.

\section{Consequences}

Let us write some remarks which follow from the above assumptions.

\section{Remark 1.}

1. (12) implies that $X$ is reflexive, moreover the operator $A_{\omega_{0}}$ is sectorial, thus $D(A)$ is dense in $X$.

2. From (13) and (14) we get for any $s \in \mathbb{R}$

$$
\sup _{s \in \mathbb{R}}\left\|e^{-\left(\theta_{A} / 2\right)|s|}\left(\sqrt{-A_{\omega_{0}}}\right)^{i s}\right\|_{\mathcal{L}(X)}<+\infty .
$$

3. By using Lemma 2.6, statement b, p. 103 in G. Dore and S. Yakubov [4], we get

$$
\left\{\begin{array}{l}
\exists K_{0}, K_{1}>0, \exists c_{0}>0 \text { such that } \forall \omega \geqslant 0,\left\|e^{2 Q_{\omega}}\right\|_{\mathcal{L}(X)} \leqslant K_{0} e^{-2 c_{0} \sqrt{\omega}} \\
\text { and }\left\|Q_{\omega} e^{2 Q_{\omega}}\right\|_{\mathcal{L}(X)} \leqslant K_{1} e^{-2 c_{0} \sqrt{\omega}}
\end{array}\right.
$$


4. Under (13), for all $\omega \geqslant \omega_{0}$, the operator $I-e^{2 Q_{\omega}}$ is boundedly invertible (see A. Lunardi [5, Corollary 2.3.7, p. 62]).

5. Suppose that problem (1), (2) has a strict solution $u$. Then, from above

$$
u \in W^{2, p}(0,1 ; X) \cap L^{p}\left(0,1 ; D\left(Q_{\omega}^{2}\right)\right), 1<p<\infty,
$$

which implies

$$
u(0), u(1) \in\left(D\left(Q_{\omega}^{2}\right), X\right)_{\frac{1}{2 p}, p}=\left(X, D\left(Q_{\omega}^{2}\right)\right)_{1-\frac{1}{2 p}, p},
$$

(see Grisvard [6, Teorema 2, p. 678]). Recall that, for $m \in \mathbb{N} \backslash\{0\}$, we have

$$
\left(D\left(Q_{\omega}^{m}\right), X\right)_{1 / m p, p}=D_{Q_{\omega}^{m}}(1-1 / m p, p),
$$

in virtue of [6]. So, by the well known reiteration Lions theorem, we get

$$
\begin{aligned}
& D_{Q_{\omega}^{m}}(1-1 / m p, p)=D_{Q_{\omega}}(m-1 / p, p)=D_{Q_{\omega}}(m-1+(1-1 / p), p)= \\
= & \left\{\varphi \in D\left(Q_{\omega}^{m-1}\right): Q_{\omega}^{m-1} \varphi \in\left(D\left(Q_{\omega}\right), X\right)_{1 / p, p}\right\}=\left(D\left(Q_{\omega}\right), X\right)_{(m-1)+1 / p, p} .
\end{aligned}
$$

In particular, for $m=2$ :

$$
\begin{gathered}
\left(D\left(Q_{\omega}^{2}\right), X\right)_{1 / 2 p, p}=D_{Q_{\omega}^{2}}(1-1 / 2 p, p)=\left(D\left(Q_{\omega}\right), X\right)_{1+1 / p, p}= \\
\quad=\left\{\varphi \in D\left(Q_{\omega}\right): Q_{\omega} \varphi \in\left(D\left(Q_{\omega}\right), X\right)_{1 / p, p}\right\} \subset D\left(Q_{\omega}\right),
\end{gathered}
$$

from which it follows that

$$
u(0), u(1) \in D\left(Q_{\omega}\right)
$$

6. Assumptions (4) and (5) involve that, for $\omega \geq \omega_{0},-A_{\omega}$ belong to the class $B I P(X, \theta)$ [7, 8, Definition 1, p. 431].

Lemma 1. Assume that (13) and (15) hold. Then there exist constants $C>0$ and $\omega_{1}>\omega_{0}$ such that, for all $\omega \geqslant \omega_{1}$ operator $Q_{\omega} \pm H$ is boundedly invertible and

$$
\left\|\left(Q_{\omega} \pm H\right)^{-1}\right\|_{\mathcal{L}(X)}<\frac{C}{\sqrt{\omega}}
$$

Proof. For all $\omega>\omega_{0}$ we have

$$
Q_{\omega}^{-1}=(-\sqrt{-(A-\omega I)})^{-1}=\frac{1}{\pi} \int_{0}^{+\infty} \frac{(A-\omega I-\mu I)^{-1}}{\mu^{1 / 2}} d \mu
$$

which implies

$$
\left\|Q_{\omega}^{-1}\right\|_{\mathcal{L}(X)} \leqslant \frac{C}{\omega^{1 / 2}}
$$

On the other hand $H Q_{\omega}^{-1}$ is well defined for all $\omega>\omega_{0}$ since, by (15), we have

$$
\begin{aligned}
\left\|H Q_{\omega}^{-1}\right\|_{\mathcal{L}(X)} & =\left\|\frac{1}{\pi} \int_{0}^{+\infty} \frac{H(A-\omega I-\mu I)^{-1}}{\mu^{1 / 2}} d \mu\right\|_{\mathcal{L}(X)} \leq \\
\leqslant C & \int_{0}^{+\infty} \frac{d \mu}{\mu^{1 / 2}|\omega+\mu|^{1 / 2+\nu}} \leqslant \frac{C}{\omega^{\nu}},
\end{aligned}
$$


so there exists $\omega_{1}>\omega_{0}$ such that for all $\omega \geqslant \omega_{1}$ we have

$$
\left\|H Q_{\omega}^{-1}\right\|_{\mathcal{L}(X)} \leqslant \frac{1}{2}
$$

which implies that operator

$$
Q_{\omega} \pm H=\left(I \pm H Q_{\omega}^{-1}\right) Q_{\omega}
$$

for $\omega \geqslant \omega_{1}$ is invertible and

$$
\begin{gathered}
\left\|\left(Q_{\omega} \pm H\right)^{-1}\right\|_{\mathcal{L}(X)}=\left\|Q_{\omega}^{-1}\left(I \pm H Q_{\omega}^{-1}\right)^{-1}\right\|_{\mathcal{L}(X)} \leq \\
\leqslant\left\|Q_{\omega}^{-1}\right\|_{\mathcal{L}(X)} \frac{1}{1-\left\|H Q_{\omega}^{-1}\right\|_{\mathcal{L}(X)}} \leqslant \frac{C}{\omega^{1 / 2}} .
\end{gathered}
$$

Lemma 2. Assume that (13) and (15) hold. Then $\left(Q_{\omega}-H\right)^{-1}$ for $\omega \geqslant \omega_{1}$ is a linear bounded operator from $\left(D\left(Q_{\omega}\right), X\right)_{\theta, q}$ into itself for all $\left.\theta \in\right] 0,1[, q \in[1, \infty]$.

Proof. Since $Q_{\omega}$ is closed then $Q_{\omega}\left(Q_{\omega} \pm H\right)^{-1}$ is bounded, whence we deduce that

$$
\left(Q_{\omega} \pm H\right)^{-1} \in \mathcal{L}\left(D\left(Q_{\omega}\right), D\left(Q_{\omega}\right)\right)
$$

(here $D\left(Q_{\omega}\right)$ is a Banach space endowed with the graph norm). So, by the well known interpolation property we get

$$
\left(Q_{\omega} \pm H\right)^{-1} \in \mathcal{L}\left(\left(D\left(Q_{\omega}\right), X\right)_{\theta, q}\right)
$$

where $\theta \in] 0,1[$ and $q \in[1, \infty]$. Therefore we deduce the result.

Remark 2. Observe that when $Q_{\omega}$ and $\left(Q_{\omega} \pm H\right)^{-1}$ are commuting for large $\omega$, the same proof as above implies that

$$
Q_{\omega}\left(Q_{\omega} \pm H\right)^{-1} \in \mathcal{L}\left(\left(D\left(Q_{\omega}\right), X\right)_{\theta, q}\right) .
$$

Therefore in this work, instead of assumption (11), we suppose

$$
Q_{\omega}\left(Q_{\omega} \pm H\right)^{-1}\left[\left(D\left(Q_{\omega}\right), X\right)_{1 / p, p}\right] \subset\left(D\left(Q_{\omega}\right), X\right)_{1 / p, p}
$$

since operators $Q_{\omega}$ and $\left(Q_{\omega} \pm H\right)^{-1}$ do not commute. This hypothesis is better in some sense than (11). We recall that

$$
\left(D\left(Q_{\omega}\right), X\right)_{1 / p, p}=\left\{x \in X: t \longmapsto t^{1-1 / p} Q_{\omega}\left(Q_{\omega}-t I\right)^{-1} x \in L_{*}^{p}\left(\mathbb{R}_{+} ; X\right)\right\},
$$

see P. Grisvard [6] and [9].

Lemma 3. Assume that (13) and (15) hold. Then there exists $\omega_{2} \geqslant \omega_{0}$ such that for $\omega \geqslant \omega_{2}$ the operator $\Lambda_{\omega}$ defined by

$$
\left\{\begin{array}{l}
D\left(\Lambda_{\omega}\right)=D\left(Q_{\omega}\right) \cap D(H)=D\left(Q_{\omega}\right) \\
\Lambda_{\omega}=\left(Q_{\omega}-H\right)+e^{2 Q_{\omega}}\left(Q_{\omega}+H\right)
\end{array}\right.
$$


is closed, boundedly invertible and

$$
\left\{\begin{array}{l}
\Lambda_{\omega}^{-1}=\left(Q_{\omega}-H\right)^{-1}(I+W) \text { with } \\
W \in \mathcal{L}(X) \text { and } W(X) \subset \bigcap_{k \in \mathbb{N} \backslash\{0\}} D\left(Q_{\omega}^{k}\right) .
\end{array}\right.
$$

Proof. Let $\omega \geqslant \omega_{0}$. In virtue of assumption (15) and Lemma 1 operator $\Lambda_{\omega}$ can be written as follows

$$
\begin{gathered}
\Lambda_{\omega}=\left(I-e^{2 Q_{\omega}}\right)\left(Q_{\omega}-H\right)+2 Q_{\omega} e^{2 Q_{\omega}}= \\
=\left(I-e^{2 Q_{\omega}}\right)\left[I+2\left(I-e^{2 Q_{\omega}}\right)^{-1} Q_{\omega} e^{2 Q_{\omega}}\left(Q_{\omega}-H\right)^{-1}\right]\left(Q_{\omega}-H\right) .
\end{gathered}
$$

Set

$$
T_{\omega}=2\left(I-e^{2 Q_{\omega}}\right)^{-1} Q_{\omega} e^{2 Q_{\omega}}\left(Q_{\omega}-H\right)^{-1} .
$$

Due to (16) and Lemma 1, we have for $\omega \geqslant \omega_{1}$

$$
\begin{gathered}
\left\|T_{\omega}\right\|_{\mathcal{L}(X)}=\left\|2\left(I-e^{2 Q_{\omega}}\right)^{-1} Q_{\omega} e^{2 Q_{\omega}}\left(Q_{\omega}-H\right)^{-1}\right\|_{\mathcal{L}(X)} \leq \\
\leq 2\left\|\left(I-e^{2 Q_{\omega}}\right)^{-1}\right\|_{\mathcal{L}(X)}\left\|Q_{\omega} e^{2 Q_{\omega}}\right\|_{\mathcal{L}(X)}\left\|\left(Q_{\omega}-H\right)^{-1}\right\|_{\mathcal{L}(X)} \leq \\
\leq \frac{2}{1-\left\|e^{2 Q_{\omega}}\right\|_{\mathcal{L}(X)}}\left\|Q_{\omega} e^{2 Q_{\omega}}\right\|_{\mathcal{L}(X)}\left\|\left(Q_{\omega}-H\right)^{-1}\right\|_{\mathcal{L}(X)} \leqslant \frac{2 C K_{1}}{\omega^{1 / 2}\left(1-K_{0} e^{-2 c_{0} \sqrt{\omega}}\right)} e^{-2 c_{0} \sqrt{\omega}}
\end{gathered}
$$

which implies the existence of $\omega_{2} \geqslant \omega_{1}$ such that for $\omega \geqslant \omega_{2}$

$$
\frac{2 C K_{1}}{\omega^{1 / 2}\left(1-K_{0} e^{-2 c_{0} \sqrt{\omega}}\right)} e^{-2 c_{0} \sqrt{\omega}}<1,
$$

hence $I+T_{\omega} \in \mathcal{L}(X)$ is boundedly invertible. Then from Remark 1, statement 4 and Lemma 1 we get that

$$
\Lambda_{\omega}=\left(I-e^{2 Q_{\omega}}\right)\left(I+T_{\omega}\right)\left(Q_{\omega}-H\right)
$$

is closed and boundedly invertible with

$$
\begin{gathered}
\Lambda_{\omega}^{-1}=\left(Q_{\omega}-H\right)^{-1}\left(I+T_{\omega}\right)^{-1}\left(I+S_{\omega}\right)^{-1}= \\
=\left(Q_{\omega}-H\right)^{-1}\left[I-T_{\omega}\left(I+T_{\omega}\right)^{-1}\right]\left[I-S_{\omega}\left(I+S_{\omega}\right)^{-1}\right] .
\end{gathered}
$$

Moreover, for $\omega \geqslant \omega_{2}$.

$$
\left\{\begin{array}{l}
T_{\omega}=2 e^{2 Q_{\omega}}\left(I-e^{2 Q_{\omega}}\right)^{-1} Q_{\omega}\left(Q_{\omega}-H\right)^{-1} \in \mathcal{L}(X) \\
S_{\omega}=-e^{2 Q_{\omega}} \in \mathcal{L}(X) \text { and } \\
T_{\omega}(X), S_{\omega}(X) \subset \bigcap_{k \in \mathbb{N} \backslash\{0\}} D\left(Q_{\omega}^{k}\right)
\end{array}\right.
$$

from which we deduce (19).

Remark 3. Assume that (13) and (15) hold. It is natural to consider for $\omega \geqslant \omega_{0}$ an operator $\Pi_{\omega}$ (instead of $\Lambda_{\omega}$ ) defined by

$$
\left\{\begin{array}{l}
D\left(\Pi_{\omega}\right)=D\left(Q_{\omega}\right) \cap D(H)=D\left(Q_{\omega}\right), \\
\Pi_{\omega}=\left(Q_{\omega}-H\right)+\left(Q_{\omega}+H\right) e^{2 Q_{\omega}},
\end{array}\right.
$$


since $e^{2 Q_{\omega}}(X) \subset D\left(Q_{\omega}\right)$. And again we obtain that for $\omega \geqslant \omega_{2}$ the operator $\Pi_{\omega}$ is closed and boundedly invertible.

Indeed, let $\omega \geqslant \omega_{0}$. The operator $\Pi_{\omega}$ can be written as follows

$$
\begin{aligned}
\Pi_{\omega} & =\left(Q_{\omega}-H\right)\left(I-e^{2 Q_{\omega}}\right)+2 Q_{\omega} e^{2 Q_{\omega}}= \\
& =\left(Q_{\omega}-H\right)\left[I+2\left(Q_{\omega}-H\right)^{-1} Q_{\omega} e^{2 Q_{\omega}}\left(I-e^{2 Q_{\omega}}\right)^{-1}\right]\left(I-e^{2 Q_{\omega}}\right),
\end{aligned}
$$

similarly to $\Lambda_{\omega}$ for $\omega \geqslant \omega_{2}$ we have

$$
\left\|2\left(Q_{\omega}-H\right)^{-1} Q_{\omega} e^{2 Q_{\omega}}\left(I-e^{2 Q_{\omega}}\right)^{-1}\right\|_{\mathcal{L}(X)}<1 .
$$

Remark 4. We can use the fact that $\left(Q_{\omega}-H\right)$ and $\left(Q_{\omega}+H\right)$ are invertible by writing

$$
\begin{aligned}
\Lambda_{\omega} & =\left(Q_{\omega}-H\right)+e^{2 Q_{\omega}}\left(Q_{\omega}+H\right)= \\
& =\left(Q_{\omega}-H\right)\left(Q_{\omega}+H\right)^{-1}\left[I+\left(Q_{\omega}+H\right)\left(Q_{\omega}-H\right)^{-1} e^{2 Q_{\omega}}\right]\left(Q_{\omega}+H\right) .
\end{aligned}
$$

Due to (16), we have

$$
\begin{gathered}
\left\|\left(Q_{\omega}+H\right)\left(Q_{\omega}-H\right)^{-1} e^{2 Q_{\omega}}\right\|_{\mathcal{L}(X)} \leq\left\|\left(Q_{\omega}+H\right)\left(Q_{\omega}-H\right)^{-1}\right\|_{\mathcal{L}(X)}\left\|e^{2 Q_{\omega}}\right\|_{\mathcal{L}(X)} \leq \\
\leq\left\|I+2 H Q_{\omega}^{-1}\left(I-H Q_{\omega}^{-1}\right)^{-1}\right\|_{\mathcal{L}(X)} K_{0} e^{-2 c_{0} \sqrt{\omega}}
\end{gathered}
$$

and due to Lemma 1 , for $\omega \geqslant \omega_{1}$ we get

$$
\begin{gathered}
\left\|2 H Q_{\omega}^{-1}\left(I-H Q_{\omega}^{-1}\right)^{-1}\right\|_{\mathcal{L}(X)} \leqslant 2\left\|H Q_{\omega}^{-1}\right\|_{\mathcal{L}(X)}\left\|\left(I-H Q_{\omega}^{-1}\right)^{-1}\right\|_{\mathcal{L}(X)} \leq \\
\leq 2\left\|H Q_{\omega}^{-1}\right\|_{\mathcal{L}(X)} \frac{1}{1-\left\|H Q_{\omega}^{-1}\right\|_{\mathcal{L}(X)}} \leqslant \frac{2 C}{\omega^{\nu}} \frac{1}{1-\frac{C}{\omega^{\nu}}},
\end{gathered}
$$

moreover, for $\omega \geqslant \omega_{2}$

$$
\frac{2 C}{\omega^{\nu}} \frac{1}{1-\frac{C}{\omega^{\nu}}}<1,
$$

whence we deduce that $\Lambda_{\omega}$ is boundedly invertible. Similarly, for $\omega \geqslant \omega_{0}$, we have

$$
\begin{aligned}
\Pi_{\omega} & =\left(Q_{\omega}-H\right)+\left(Q_{\omega}+H\right) e^{2 Q_{\omega}}= \\
& =\left[I+\left(Q_{\omega}+H\right) e^{2 Q_{\omega}}\left(Q_{\omega}-H\right)^{-1}\right]\left(Q_{\omega}-H\right),
\end{aligned}
$$

and similarly to $\Lambda_{\omega}$ for $\omega \geqslant \omega_{2}$ we obtain

$$
\begin{gathered}
\left\|\left(Q_{\omega}+H\right) e^{2 Q_{\omega}}\left(Q_{\omega}-H\right)^{-1}\right\|_{\mathcal{L}(X)}= \\
=\left\|\left(I+H Q_{\omega}^{-1}\right) e^{2 Q_{\omega}}\left(I-H Q_{\omega}^{-1}\right)^{-1}\right\|_{\mathcal{L}(X)}<1 .
\end{gathered}
$$

Let us compare $\Lambda_{\omega}$ and $\Pi_{\omega}$. First we have $D\left(\Lambda_{\omega}\right)=D\left(\Pi_{\omega}\right)=D\left(Q_{\omega}\right)$, moreover

$$
\begin{gathered}
\Pi_{\omega}=Q_{\omega}-H+\left(Q_{\omega}+H\right) e^{2 Q_{\omega}}=\Lambda_{\omega}-e^{2 Q_{\omega}}\left(Q_{\omega}+H\right)+\left(Q_{\omega}+H\right) e^{2 Q_{\omega}}= \\
=\Lambda_{\omega}-e^{2 Q_{\omega}} H+H e^{2 Q_{\omega}}=\Lambda_{\omega}+\left[H ; e^{2 Q_{\omega}}\right]
\end{gathered}
$$


where, for all $\xi \in D(H)=D\left(Q_{\omega}\right)$

$$
\left[H ; e^{2 Q_{\omega}}\right] \xi=H e^{2 Q_{\omega}} \xi-e^{2 Q_{\omega}} H \xi .
$$

Then

$$
\Pi_{\omega}=\left(I+\left[H ; e^{2 Q_{\omega}}\right] \Lambda_{\omega}^{-1}\right) \Lambda_{\omega}
$$

Using (20) and due to Lemma 1 , for $\omega \geqslant \omega_{2}$ we have

$$
\begin{aligned}
\left\|\Lambda_{\omega}^{-1}\right\|_{\mathcal{L}(X)} & \leq\left\|\left(Q_{\omega}-H\right)^{-1}\right\|_{\mathcal{L}(X)}\left\|\left(I+T_{\omega}\right)^{-1}\right\|_{\mathcal{L}(X)}\left\|\left(I+S_{\omega}\right)^{-1}\right\|_{\mathcal{L}(X)} \leq \\
& \leq\left\|\left(Q_{\omega}-H\right)^{-1}\right\|_{\mathcal{L}(X)} \frac{1}{1-\left\|T_{\omega}\right\|_{\mathcal{L}(X)}} \frac{1}{1-\left\|S_{\omega}\right\|_{\mathcal{L}(X)}} \leq \\
& \leq\left\|\left(Q_{\omega}-H\right)^{-1}\right\|_{\mathcal{L}(X)} \frac{1}{1-\frac{2 C K_{1}}{\omega^{1 / 2}\left(1-K_{0} e^{-2 c_{0} \sqrt{\omega}}\right)} e^{-2 c_{0} \sqrt{\omega}}} \frac{1}{1-K_{0} e^{-2 c_{0} \sqrt{\omega}}} \leq \\
& \leq\left\|\left(Q_{\omega}-H\right)^{-1}\right\|_{\mathcal{L}(X)} \leq \frac{C}{\omega^{1 / 2}} .
\end{aligned}
$$

Due to (16), we get

$$
\begin{gathered}
\left\|H e^{2 Q_{\omega}} \Lambda_{\omega}^{-1}\right\|_{\mathcal{L}(X)} \leq\left\|H Q_{\omega}^{-1}\right\|_{\mathcal{L}(X)}\left\|Q_{\omega} e^{2 Q_{\omega}}\right\|_{\mathcal{L}(X)}\left\|\Lambda_{\omega}^{-1}\right\|_{\mathcal{L}(X)} \leq \\
\leq C \frac{1}{\omega^{\nu}} K_{1} e^{-2 c_{0} \sqrt{\omega}} \frac{1}{\omega^{1 / 2}} \leq \frac{C}{\omega^{\nu+1 / 2}} .
\end{gathered}
$$

In the other hand,

$$
\begin{gathered}
\left\|e^{2 Q_{\omega}} H \Lambda_{\omega}^{-1}\right\|_{\mathcal{L}(X)} \leq \\
\leq\left\|e^{2 Q_{\omega}}\right\|_{\mathcal{L}(X)}\left\|H\left(Q_{\omega}-H\right)^{-1}\right\|_{\mathcal{L}(X)}\left\|\left(I+T_{\omega}\right)^{-1}\right\|_{\mathcal{L}(X)}\left\|\left(I+S_{\omega}\right)^{-1}\right\|_{\mathcal{L}(X)} \leq \\
\leq K_{0} e^{-2 c_{0} \sqrt{\omega}}\left\|H\left(Q_{\omega}-H\right)^{-1}\right\|_{\mathcal{L}(X)} \leq K_{0} e^{-2 c_{0} \sqrt{\omega}}\left\|H Q_{\omega}^{-1}\right\|_{\mathcal{L}(X)}\left\|\left(I-H Q_{\omega}^{-1}\right)^{-1}\right\|_{\mathcal{L}(X)} \leq \\
\leq C K_{0} e^{-2 c_{0} \sqrt{\omega}} \frac{1}{\omega^{\nu}} \frac{1}{1-\left\|H Q_{\omega}^{-1}\right\|_{\mathcal{L}(X)}} \leq C K_{0} e^{-2 c_{0} \sqrt{\omega}} \frac{1}{\omega^{\nu}} \leq \frac{C}{\omega^{\nu}} .
\end{gathered}
$$

Hence

$$
\left\|\left[H ; e^{2 Q_{\omega}}\right] \Lambda_{\omega}^{-1}\right\|_{\mathcal{L}(X)} \leq \frac{C}{\omega^{\nu}}
$$

so there exists $\omega_{3} \geqslant \omega_{2}$ such that for all $\omega \geqslant \omega_{3}$ we have

$$
\frac{C}{\omega^{\nu}} \leq 1
$$

which confirm the invertibility of $\Pi_{\omega}$ whith

$$
\Pi_{\omega}^{-1}=\Lambda_{\omega}^{-1}\left(I+\left[H ; e^{2 Q_{\omega}}\right] \Lambda_{\omega}^{-1}\right)^{-1}
$$

Similarly, due to $(22)$, we obtain

$$
\Lambda_{\omega}^{-1}=\Pi_{\omega}^{-1}\left(I-\left[H ; e^{2 Q_{\omega}}\right] \Pi_{\omega}^{-1}\right)^{-1}
$$

Lemma 4. [10, Theorem, p. 96] Assume that (13) holds. Let $p \in] 1,+\infty[, \varphi \in X$ and $n \in \mathbb{N}^{*}$. Then, we have 
1. $e^{Q_{\omega}} \varphi \in L^{p}(0,1, X)$,

2. $Q_{\omega}^{n} e^{\cdot Q_{\omega}} \varphi \in L^{p}(0,1, X)$ if and only if $\varphi \in\left(D\left(Q_{\omega}^{n}\right), X\right)_{\frac{1}{n p}, p}$.

Lemma 5. Assume that (12) - (14) hold. Then for $f \in L^{p}(0,1, X)$ with $1<p<+\infty$, we have

1. $x \rightarrow L(x, f)=Q_{\omega} \int_{0}^{x} e^{(x-s) Q_{\omega}} f(s) d s \in L^{p}(0,1, X)$,

2. $x \rightarrow L(1-x, f(1-))=.Q_{\omega} \int_{x}^{1} e^{(s-x) Q_{\omega}} f(s) d s \in L^{p}(0,1, X)$,

3. $x \rightarrow \mathcal{L}(x, f)=Q_{\omega} \int_{0}^{1} e^{(x+s) Q_{\omega}} f(s) d s \in L^{p}(0,1, X)$,

4. $\int_{0}^{1} e^{s Q_{\omega}} f(s) d s, \int_{0}^{1} e^{(1-s) Q_{\omega}} f(s) d s \in\left(D\left(Q_{\omega}\right), X\right)_{\frac{1}{p}, p}$.

Proof. For statements 1, 2 and 3, see [3, 11, 12, pp. 167, 168], and also [13, (24), (25) and (26)]. Statement 4 is an easy consequence of statements 1 and 2, we proceed as in Remark 1 by using the fact that $x \rightarrow \int_{0}^{x} e^{(x-s) Q_{\omega}} f(s) d s$ and $x \rightarrow \int_{x}^{1} e^{(s-x) Q_{\omega}} f(s) d s$ belong to $W^{1, p}(0,1, X) \cap L^{p}\left(0,1, D\left(Q_{\omega}\right)\right)$.

\section{Representation of the Solution}

Assume that (13) and (15) hold. Consider for a.e. $x \in(0,1)$ the following formula

$$
\begin{gathered}
u(x)=\left(e^{x Q_{\omega}}-e^{(2-x) Q_{\omega}}\right) \Lambda_{\omega}^{-1} d_{0}+e^{(1-x) Q_{\omega}} u_{1}+ \\
+Q_{\omega}^{-1}\left(e^{x Q_{\omega}}-e^{(2-x) Q_{\omega}}\right)\left(Q_{\omega}+H\right) \Lambda_{\omega}^{-1} Q_{\omega} e^{Q_{\omega}} u_{1}+ \\
+\frac{1}{2} Q_{\omega}^{-1}\left(e^{x Q_{\omega}}-e^{(2-x) Q_{\omega}}\right)\left(Q_{\omega}+H\right) \Lambda_{\omega}^{-1} \int_{0}^{1}\left(e^{s Q_{\omega}}-e^{(2-s) Q_{\omega}}\right) f(s) d s- \\
-\frac{1}{2} e^{(1-x) Q_{\omega}} Q_{\omega}^{-1} \int_{0}^{1} e^{(1-s) Q_{\omega}} f(s) d s+\frac{1}{2} Q_{\omega}^{-1} \int_{0}^{x} e^{(x-s) Q_{\omega}} f(s) d s+\frac{1}{2} Q_{\omega}^{-1} \int_{x}^{1} e^{(s-x) Q_{\omega}} f(s) d s,
\end{gathered}
$$

used in Cheggag et al [1] in the commutative case.

The main idea is in searching a solution $u$ to (1), (2) for a.e. $x \in(0,1)$, in the following form:

$$
\begin{gathered}
u(x)=\left(e^{x Q_{\omega}}-e^{(2-x) Q_{\omega}}\right) \Lambda_{\omega}^{-1} d_{0}^{*}+e^{(1-x) Q_{\omega}} u_{1}^{*}+ \\
+Q_{\omega}^{-1}\left(e^{x Q_{\omega}}-e^{(2-x) Q_{\omega}}\right)\left(Q_{\omega}+H\right) \Lambda_{\omega}^{-1} Q_{\omega} e^{Q_{\omega}} u_{1}^{*}+ \\
+\frac{1}{2} Q_{\omega}^{-1}\left(e^{x Q_{\omega}}-e^{(2-x) Q_{\omega}}\right)\left(Q_{\omega}+H\right) \Lambda_{\omega}^{-1} \int_{0}^{1}\left(e^{s Q_{\omega}}-e^{(2-s) Q_{\omega}}\right) f^{*}(s) d s- \\
-\frac{1}{2} Q_{\omega}^{-1} e^{(1-x) Q_{\omega}} \int_{0}^{1} e^{(1-s) Q_{\omega}} f^{*}(s) d s+\frac{1}{2} Q_{\omega}^{-1} \int_{0}^{x} e^{(x-s) Q_{\omega}} f^{*}(s) d s+\frac{1}{2} Q_{\omega}^{-1} \int_{x}^{1} e^{(s-x) Q_{\omega}} f^{*}(s) d s .
\end{gathered}
$$

Taking into account the boundary conditions (2) deduce $d_{0}^{*}, u_{1}^{*}$ and $f^{*}$. 
It is easy to obtain

$$
u_{1}^{*}=u(1)=u_{1} .
$$

Now, from (25) for a.e. $x \in(0,1)$, we have

$$
\begin{gathered}
u^{\prime}(x)=Q_{\omega}\left(e^{x Q_{\omega}}+e^{(2-x) Q_{\omega}}\right) \Lambda_{\omega}^{-1} d_{0}^{*}-Q_{\omega} e^{(1-x) Q_{\omega}} u_{1}+ \\
+\left(e^{x Q_{\omega}}+e^{(2-x) Q_{\omega}}\right)\left(Q_{\omega}+H\right) \Lambda_{\omega}^{-1} Q_{\omega} e^{Q_{\omega}} u_{1}+ \\
+\frac{1}{2}\left(e^{x Q_{\omega}}+e^{(2-x) Q_{\omega}}\right)\left(Q_{\omega}+H\right) \Lambda_{\omega}^{-1} \int_{0}^{1}\left(e^{s Q_{\omega}}-e^{(2-s) Q_{\omega}}\right) f^{*}(s) d s+ \\
+\frac{1}{2} e^{(1-x) Q_{\omega}} \int_{0}^{1} e^{(1-s) Q_{\omega}} f^{*}(s) d s+\frac{1}{2} \int_{0}^{x} e^{(x-s) Q_{\omega}} f^{*}(s) d s-\frac{1}{2} \int_{x}^{1} e^{(s-x) Q_{\omega}} f^{*}(s) d s,
\end{gathered}
$$

and

$$
\begin{gathered}
u^{\prime \prime}(x)=Q_{\omega}^{2}\left(e^{x Q_{\omega}}-e^{(2-x) Q_{\omega}}\right) \Lambda_{\omega}^{-1} d_{0}^{*}+Q_{\omega}^{2} e^{(1-x) Q_{\omega}} u_{1}+ \\
+Q_{\omega}\left(e^{x Q_{\omega}}-e^{(2-x) Q_{\omega}}\right)\left(Q_{\omega}+H\right) \Lambda_{\omega}^{-1} Q_{\omega} e^{Q_{\omega}} u_{1}+ \\
+\frac{1}{2} Q_{\omega}\left(e^{x Q_{\omega}}-e^{(2-x) Q_{\omega}}\right)\left(Q_{\omega}+H\right) \Lambda_{\omega}^{-1} \int_{0}^{1}\left(e^{s Q_{\omega}}-e^{(2-s) Q_{\omega}}\right) f^{*}(s) d s- \\
-\frac{1}{2} Q_{\omega} e^{(1-x) Q_{\omega}} \int_{0}^{1} e^{(1-s) Q_{\omega}} f^{*}(s) d s+\frac{1}{2} Q_{\omega} \int_{0}^{x} e^{(x-s) Q_{\omega}} f^{*}(s) d s+ \\
+\frac{1}{2} Q_{\omega} \int_{x}^{1} e^{(s-x) Q_{\omega}} f^{*}(s) d s+f^{*}(x) .
\end{gathered}
$$

Since $A_{\omega}=-Q_{\omega}^{2}$ and in virtue of (25), it is easy to see that

$$
u^{\prime \prime}(x)+A_{\omega} u(x)=f^{*}(x),
$$

therefore, for a.e. $x \in(0,1)$, we deduce

$$
f^{*}(x)=f(x) .
$$

Now, for $d_{0}^{*}$ we have

$$
\begin{gathered}
u(0)=\left(I-e^{2 Q_{\omega}}\right) \Lambda_{\omega}^{-1} d_{0}^{*}+Q_{\omega}^{-1}\left[I+\left(I-e^{2 Q_{\omega}}\right)\left(Q_{\omega}+H\right) \Lambda_{\omega}^{-1}\right] Q_{\omega} e^{Q_{\omega}} u_{1}+ \\
+\frac{1}{2} Q_{\omega}^{-1}\left[I+\left(I-e^{2 Q_{\omega}}\right)\left(Q_{\omega}+H\right) \Lambda_{\omega}^{-1}\right] \int_{0}^{1}\left(e^{s Q_{\omega}}-e^{(2-s) Q_{\omega}}\right) f(s) d s
\end{gathered}
$$

Note that

$$
\begin{gathered}
I+\left(I-e^{2 Q_{\omega}}\right)\left(Q_{\omega}+H\right) \Lambda_{\omega}^{-1}=\left[\Lambda_{\omega}+Q_{\omega}+H-e^{2 Q_{\omega}}\left(Q_{\omega}+H\right)\right] \Lambda_{\omega}^{-1}= \\
=\left[Q_{\omega}-H+e^{2 Q_{\omega}}\left(Q_{\omega}+H\right)+Q_{\omega}+H-e^{2 Q_{\omega}}\left(Q_{\omega}+H\right)\right] \Lambda_{\omega}^{-1}=2 Q_{\omega} \Lambda_{\omega}^{-1},
\end{gathered}
$$

then

$$
u(0)=\left(I-e^{2 Q_{\omega}}\right) \Lambda_{\omega}^{-1} d_{0}^{*}+2 \Lambda_{\omega}^{-1} Q_{\omega} e^{Q_{\omega}} u_{1}+\Lambda_{\omega}^{-1} \int_{0}^{1}\left(e^{s Q_{\omega}}-e^{(2-s) Q_{\omega}}\right) f(s) d s .
$$

In virtue of assumption (15) and due to Lemma 3, we deduce that $u(0) \in D(H)$. Applying $H$ to $(30)$, we get

$$
H u(0)=H\left(I-e^{2 Q_{\omega}}\right) \Lambda_{\omega}^{-1} d_{0}^{*}+2 H \Lambda_{\omega}^{-1} Q_{\omega} e^{Q_{\omega}} u_{1}+H \Lambda_{\omega}^{-1} \int_{0}^{1}\left(e^{s Q_{\omega}}-e^{(2-s) Q_{\omega}}\right) f(s) d s .
$$


Using (27) for $x=0$, we obtain

$$
\begin{aligned}
u^{\prime}(0) & =Q_{\omega}\left(I+e^{2 Q_{\omega}}\right) \Lambda_{\omega}^{-1} d_{0}^{*}+\left[\left(I+e^{2 Q_{\omega}}\right)\left(Q_{\omega}+H\right) \Lambda_{\omega}^{-1}-I\right] Q_{\omega} e^{Q_{\omega}} u_{1}+ \\
& +\frac{1}{2}\left[\left(I+e^{2 Q_{\omega}}\right)\left(Q_{\omega}+H\right) \Lambda_{\omega}^{-1}-I\right] \int_{0}^{1}\left(e^{s Q_{\omega}}-e^{(2-s) Q_{\omega}}\right) f(s) d s
\end{aligned}
$$

On the other hand, we have

$$
\begin{aligned}
& \left(I+e^{2 Q_{\omega}}\right)\left(Q_{\omega}+H\right) \Lambda_{\omega}^{-1}-I=\left[Q_{\omega}+H+e^{2 Q_{\omega}}\left(Q_{\omega}+H\right)-\Lambda_{\omega}\right] \Lambda_{\omega}^{-1}= \\
= & {\left[Q_{\omega}+H+e^{2 Q_{\omega}}\left(Q_{\omega}+H\right)-\left(Q_{\omega}-H\right)-e^{2 Q_{\omega}}\left(Q_{\omega}+H\right)\right] \Lambda_{\omega}^{-1}=2 H \Lambda_{\omega}^{-1} . }
\end{aligned}
$$

Then we get

$$
\begin{aligned}
u^{\prime}(0)= & Q_{\omega}\left(I+e^{2 Q_{\omega}}\right) \Lambda_{\omega}^{-1} d_{0}^{*}+2 H \Lambda_{\omega}^{-1} Q_{\omega} e^{Q_{\omega}} u_{1}+ \\
& +H \Lambda_{\omega}^{-1} \int_{0}^{1}\left(e^{s Q_{\omega}}-e^{(2-s) Q_{\omega}}\right) f(s) d s .
\end{aligned}
$$

Therefore, using (31) and (32), we conclude that

$$
u^{\prime}(0)-H u(0)=\left[Q_{\omega}\left(I+e^{2 Q_{\omega}}\right)-H\left(I-e^{2 Q_{\omega}}\right)\right] \Lambda_{\omega}^{-1} d_{0}^{*}=\Pi_{\omega} \Lambda_{\omega}^{-1} d_{0}^{*}=d_{0} .
$$

Then, due to Remak 3 and (23), we obtain

$$
d_{0}^{*}=\Lambda_{\omega} \Pi_{\omega}^{-1} d_{0}=\left(I+\left[H ; e^{2 Q_{\omega}}\right] \Lambda_{\omega}^{-1}\right)^{-1} d_{0} .
$$

Finally, inserting (33) into (25), from (26) and (29) we deduce that $u$ is given formally by

$$
\begin{gathered}
u(x)=\left(e^{x Q_{\omega}}-e^{(2-x) Q_{\omega}}\right) \Lambda_{\omega}^{-1}\left(I+\left[H ; e^{2 Q_{\omega}}\right] \Lambda_{\omega}^{-1}\right)^{-1} d_{0}+e^{(1-x) Q_{\omega}} u_{1}+ \\
+Q_{\omega}^{-1}\left(e^{x Q_{\omega}}-e^{(2-x) Q_{\omega}}\right)\left(Q_{\omega}+H\right) \Lambda_{\omega}^{-1} Q_{\omega} e^{Q_{\omega}} u_{1}+ \\
+\frac{1}{2} Q_{\omega}^{-1}\left(e^{x Q_{\omega}}-e^{(2-x) Q_{\omega}}\right)\left(Q_{\omega}+H\right) \Lambda_{\omega}^{-1} \int_{0}^{1}\left(e^{s Q_{\omega}}-e^{(2-s) Q_{\omega}}\right) f(s) d s- \\
-\frac{1}{2} Q_{\omega}^{-1} e^{(1-x) Q_{\omega}} \int_{0}^{1} e^{(1-s) Q_{\omega}} f(s) d s+\frac{1}{2} Q_{\omega}^{-1} \int_{0}^{x} e^{(x-s) Q_{\omega}} f(s) d s+\frac{1}{2} Q_{\omega}^{-1} \int_{x}^{1} e^{(s-x) Q_{\omega}} f(s) d s .
\end{gathered}
$$

Note that if the commutativity hypothesis $(7)$ is verified then the commutator $\left[H ; e^{2 Q_{\omega}}\right]$ becomes zero and then the representation formulas of solution (10) and (39) coincide.

Now we can write $u$ as

$$
u(\cdot)=S_{1}\left(\cdot, d_{0}, u_{1}\right)+S_{2}\left(\cdot, u_{1}, f\right)+D(\cdot, f)-R\left(\cdot, d_{0}, u_{1}, f\right),
$$

where, for a.e. $x \in(0,1)$

$$
\begin{gathered}
S_{1}\left(x, d_{0}, u_{1}\right)=e^{x Q_{\omega}} \Lambda_{\omega}^{-1}\left(I+\left[H ; e^{2 Q_{\omega}}\right] \Lambda_{\omega}^{-1}\right)^{-1} d_{0}+e^{(1-x) Q_{\omega}} u_{1}, \\
S_{2}\left(x, u_{1}, f\right)=Q_{\omega}^{-1} e^{x Q_{\omega}}\left(Q_{\omega}+H\right) \Lambda_{\omega}^{-1} Q_{\omega} e^{Q_{\omega}} u_{1}+ \\
+\frac{1}{2} Q_{\omega}^{-1} e^{x Q_{\omega}}\left(Q_{\omega}+H\right) \Lambda_{\omega}^{-1} \int_{0}^{1}\left(e^{s Q_{\omega}}-e^{(2-s) Q_{\omega}}\right) f(s) d s \\
D(x, f)=-\frac{1}{2} e^{(1-x) Q_{\omega}} Q_{\omega}^{-1} \int_{0}^{1} e^{(1-s) Q_{\omega}} f(s) d s+\frac{1}{2} Q_{\omega}^{-1} \int_{0}^{x} e^{(x-s) Q_{\omega}} f(s) d s+ \\
+\frac{1}{2} Q_{\omega}^{-1} \int_{x}^{1} e^{(s-x) Q_{\omega}} f(s) d s
\end{gathered}
$$


and

$$
\begin{gathered}
R\left(x, d_{0}, u_{1}, f\right)=e^{(2-x) Q_{\omega}} \Lambda_{\omega}^{-1}\left(I+\left[H ; e^{2 Q_{\omega}}\right] \Lambda_{\omega}^{-1}\right)^{-1} d_{0}+ \\
+e^{(2-x) Q_{\omega}} Q_{\omega}^{-1}\left(Q_{\omega}+H\right) \Lambda_{\omega}^{-1} Q_{\omega} e^{Q_{\omega}} u_{1}+ \\
+\frac{1}{2} e^{(2-x) Q_{\omega}} Q_{\omega}^{-1}\left(Q_{\omega}+H\right) \Lambda_{\omega}^{-1} \int_{0}^{1}\left(e^{s Q_{\omega}}-e^{(2-s) Q_{\omega}}\right) f(s) d s .
\end{gathered}
$$

On the other hand, due to (24), we have

$$
\begin{gathered}
u(x)=\left(e^{x Q_{\omega}}-e^{(2-x) Q_{\omega}}\right) \Pi_{\omega}^{-1} d_{0}+e^{(1-x) Q_{\omega}} u_{1}+ \\
+Q_{\omega}^{-1}\left(e^{x Q_{\omega}}-e^{(2-x) Q_{\omega}}\right)\left(Q_{\omega}+H\right) \Pi_{\omega}^{-1}\left(I-\left[H ; e^{2 Q_{\omega}}\right] \Pi_{\omega}^{-1}\right)^{-1} Q_{\omega} e^{Q_{\omega}} u_{1}+ \\
+\frac{1}{2} Q_{\omega}^{-1}\left(e^{x Q_{\omega}}-e^{(2-x) Q_{\omega}}\right)\left(Q_{\omega}+H\right) \Pi_{\omega}^{-1}\left(I-\left[H ; e^{2 Q_{\omega}}\right] \Pi_{\omega}^{-1}\right)^{-1} \int_{0}^{1}\left(e^{s Q_{\omega}}-e^{(2-s) Q_{\omega}}\right) f(s) d s+ \\
-\frac{1}{2} Q_{\omega}^{-1} e^{(1-x) Q_{\omega}} \int_{0}^{1} e^{(1-s) Q_{\omega}} f(s) d s+\frac{1}{2} Q_{\omega}^{-1} \int_{0}^{x} e^{(x-s) Q_{\omega}} f(s) d s+\frac{1}{2} Q_{\omega}^{-1} \int_{x}^{1} e^{(s-x) Q_{\omega}} f(s) d s .
\end{gathered}
$$

\section{Main Result}

Under assumptions (12) - (15) and (18), we focus on the study of the optimal regularity of the strict solution given by (39).

Lemma 6. Assume that (13), (15) and (18) hold. For any $\left.\varphi \in\left(D\left(Q_{\omega}\right), X\right)_{\frac{1}{p}, p}, p \in\right] 0, \infty[$ and $\omega \geqslant \omega_{2}$, we have

$$
\left(Q_{\omega} \pm H\right) \Lambda_{\omega}^{-1} \varphi \in\left(D\left(Q_{\omega}\right), X\right)_{\frac{1}{p}, p}
$$

Proof. We have a representation

$$
\left(Q_{\omega}+H\right) \Lambda_{\omega}^{-1}=2 Q_{\omega} \Lambda_{\omega}^{-1}+e^{2 Q_{\omega}}\left(Q_{\omega}+H\right) \Lambda_{\omega}^{-1}-I,
$$

which, by Lemma 3, can be written as follows

$$
\left(Q_{\omega}+H\right) \Lambda_{\omega}^{-1}=2 Q_{\omega}\left(Q_{\omega}-H\right)^{-1}(I+W)+e^{2 Q_{\omega}}\left(Q_{\omega}+H\right) \Lambda_{\omega}^{-1}-I,
$$

where $W(X) \subset \bigcap_{k \in \mathbb{N} \backslash\{0\}} D\left(Q_{\omega}^{k}\right) \subset\left(D\left(Q_{\omega}\right), X\right)_{\frac{1}{p}, p}$.

Then, due to assumption (18), for any $\varphi \in\left(D\left(Q_{\omega}\right), X\right)_{\frac{1}{p}, p}$, we deduce that $\left(Q_{\omega}+H\right) \Lambda_{\omega}^{-1} \varphi \in\left(D\left(Q_{\omega}\right), X\right)_{\frac{1}{p}, p}$.

On the other hand, we also have

$$
\left(Q_{\omega}-H\right) \Lambda_{\omega}^{-1}=I-e^{2 Q_{\omega}}\left(Q_{\omega}+H\right) \Lambda_{\omega}^{-1}
$$

Thus, for any $\varphi \in\left(D\left(Q_{\omega}\right), X\right)_{\frac{1}{p}, p}$, we get $\left(Q_{\omega}-H\right) \Lambda_{\omega}^{-1} \varphi \in\left(D\left(Q_{\omega}\right), X\right)_{\frac{1}{p}, p}$.

Lemma 7. Assume that (13) and (15) hold. Let $d_{0}, u_{1} \in X, \omega \geqslant \omega_{2}$ and $f \in$ $L^{p}(0,1 ; X), 1<p<\infty$. Then

$$
A_{\omega} R\left(\cdot, d_{0}, u_{1}, f\right) \in L^{p}(0,1 ; X) .
$$


Proof. Since $A_{\omega}=-Q_{\omega}^{2}, e^{(2-\cdot) Q_{\omega}}=e^{Q_{\omega}} e^{(1-\cdot) Q_{\omega}}$ and $e^{Q_{\omega}} \xi \in \bigcap_{k=1}^{\infty} D\left(Q_{\omega}^{k}\right)$ for all $\xi \in X$, taking into account (38), the term

$$
\begin{gathered}
A_{\omega} R\left(x, d_{0}, u_{1}, f\right)=-Q_{\omega} e^{(2-x) Q_{\omega}} Q_{\omega} \Lambda_{\omega}^{-1}\left(I+\left[H ; e^{2 Q_{\omega}}\right] \Lambda_{\omega}^{-1}\right)^{-1} d_{0}- \\
-Q_{\omega} e^{(2-x) Q_{\omega}}\left(Q_{\omega}+H\right) \Lambda_{\omega}^{-1} Q_{\omega} e^{Q_{\omega}} u_{1}- \\
-\frac{1}{2} Q_{\omega} e^{(2-x) Q_{\omega}}\left(Q_{\omega}+H\right) \Lambda_{\omega}^{-1} \int_{0}^{1}\left(e^{s Q_{\omega}}-e^{(2-s) Q_{\omega}}\right) f(s) d s=Q_{\omega} e^{Q_{\omega}} e^{(1-x) Q_{\omega}} \xi
\end{gathered}
$$

is bounded and thus belongs to $L^{p}(0,1 ; X), 1<p<\infty$ for all $\xi \in X$.

Remark 5. As we have seen, the term $R\left(\cdot, d_{0}, u_{1}, f\right)$ is regular, but this can not be applied to the term $e^{\cdot Q_{\omega}}\left(Q_{\omega}+H\right) \Lambda_{\omega}^{-1} e^{Q_{\omega}} Q_{\omega} u_{1}$ in $S_{2}\left(\cdot, u_{1}, f\right)$ since operators $\left(Q_{\omega}+H\right) \Lambda_{\omega}^{-1}$ and $e^{Q_{\omega}}$ are not considered to be commutative.

Remark 6. Assume that (12) - (14) hold and $f \in L^{p}(0,1 ; X)$ with $1<p<\infty$. Then, by using Lemma 5 , we can easily see that

$$
A_{\omega} D(\cdot, f) \in L^{p}(0,1 ; X)
$$

see for instance [1].

Lemma 8. Assume that (12) - (15) hold and (18) and $f \in L^{p}(0,1 ; X), 1<p<\infty$. Then, for all $\omega \geqslant \omega_{2}$

$$
A_{\omega} S_{2}\left(., u_{1}, f\right) \in L^{p}(0,1 ; X), 1<p<\infty
$$

Proof. Taking into account $(36)$ and $A_{\omega}=-Q_{\omega}^{2}$, for a.e. $x \in(0,1)$ we can write

$$
\begin{aligned}
A_{\omega} S_{2}\left(x, u_{1}, f\right)= & -Q_{\omega} e^{x Q_{\omega}}\left(Q_{\omega}+H\right) \Lambda_{\omega}^{-1} e^{Q_{\omega}} Q_{\omega} u_{1}- \\
& -\frac{1}{2} Q_{\omega} e^{x Q_{\omega}}\left(Q_{\omega}+H\right) \Lambda_{\omega}^{-1} \int_{0}^{1}\left(e^{s Q_{\omega}}-e^{(2-s) Q_{\omega}}\right) f(s) d s .
\end{aligned}
$$

Since

$$
e^{Q_{\omega}} Q_{\omega} u_{1}, \int_{0}^{1}\left(e^{s Q_{\omega}}-e^{(2-s) Q_{\omega}}\right) f(s) d s \in\left(D\left(Q_{\omega}\right), X\right)_{\frac{1}{p}, p},
$$

then, due to Lemmas 4 and 6 , we deduce that

$$
A S_{2}(., f) \in L^{p}(0,1, X), 1<p<\infty .
$$

Theorem 2. Assume that (12) - (15) and (18) hold. Let $f \in L^{p}(0,1 ; X)$ with $1<p<\infty$ and $\omega \geqslant \omega_{2}$. Then, the following assertions are equivalent

1. Problem (1), (2) has a unique strict solution $u$, that is

$$
u \in W^{2, p}(0,1 ; X) \cap L^{p}(0,1 ; D(A)), 1<p<\infty,
$$

and $u$ satisfies (1), (2). 
2. $\Pi_{\omega}^{-1} d_{0}, u_{1} \in(D(A), X)_{\frac{1}{2 p}, p}$ where

$$
\Pi_{\omega}=Q_{\omega}-H+\left(Q_{\omega}+H\right) e^{2 Q_{\omega}} .
$$

Proof. a) Let us begin with a uniqueness result. Let $u, \widetilde{u}$ be strict solutions of (1), (2), then $v=u-\widetilde{u}$ is a strict solution of

$$
\left\{\begin{array}{l}
v^{\prime \prime}(x)+A_{\omega} v(x)=0, \quad \text { a.e. } x \in(0,1) \\
v^{\prime}(0)-H v(0)=0, v(1)=0
\end{array}\right.
$$

Then $v \in C^{1}([0,1] ; X)$ and there exist $y_{0}, z_{1} \in D\left(Q_{\omega}\right)$ such that, for any $x \in[0,1]$

$$
v(x)=e^{x Q_{\omega}} y_{0}+e^{(1-x) Q_{\omega}} z_{1},
$$

see Section 3, in [1]. Moreover, for any $x \in[0,1]$

$$
v^{\prime}(x)=Q_{\omega} e^{x Q_{\omega}} y_{0}-Q_{\omega} e^{(1-x) Q_{\omega}} z_{1}
$$

Note that $v(0)=y_{0}+e^{Q_{\omega}} z_{1} \in D\left(Q_{\omega}\right) \cap D(H)$ and using the boundary conditions (2), we get

$$
\left\{\begin{array}{l}
v^{\prime}(0)-H v(0)=\left(Q_{\omega} y_{0}-Q_{\omega} e^{Q_{\omega}} z_{1}\right)-H\left(y_{0}+e^{Q_{\omega}} z_{1}\right)=0 \\
v(1)=e^{Q_{\omega}} y_{0}+z_{1}=0
\end{array}\right.
$$

From $z_{1}=-e^{Q_{\omega}} y_{0}$ we deduce

$$
\begin{aligned}
& 0=\left(Q_{\omega} y_{0}+Q_{\omega} e^{2 Q_{\omega}} y_{0}\right)-H\left(y_{0}-e^{2 Q_{\omega}} y_{0}\right)= \\
& =\left[Q_{\omega}\left(I+e^{2 Q_{\omega}}\right)-H\left(I-e^{2 Q_{\omega}}\right)\right] y_{0}=\prod_{\omega} y_{0},
\end{aligned}
$$

and due to Remark 3 , for all $\omega \geqslant \omega_{2}$ we get $y_{0}=0$ and then $z_{1}=0$. So, $v=0$ and thus $u=\widetilde{u}$.

b) Consider $u$ given by (39). Let us show that $A_{\omega} u \in L^{p}(0,1 ; X)$ with $1<p<$ $\infty$. In fact, due to Lemmas 7 and 8 and from Remark (6), it is enough to prove that $A_{\omega} S_{1}\left(., d_{0}, u_{1}\right) \in L^{p}(0,1 ; X)$. From $(35)$ for almost every $x \in(0,1)$ one has

$$
A_{\omega} S_{1}\left(x, d_{0}, u_{1}\right)=-Q_{\omega}^{2} e^{x Q_{\omega}} \Pi_{\omega}^{-1} d_{0}-Q_{\omega}^{2} e^{(1-x) Q_{\omega}} u_{1}
$$

From (17), we have $u_{1} \in\left(D\left(Q_{\omega}^{2}\right), X\right)_{\frac{1}{2 p}, p}$ and thus, due to Lemma 4 , statement 2 we get

$$
Q_{\omega}^{2} e^{(1-\cdot) Q_{\omega}} u_{1} \in L^{p}(0,1 ; X), 1<p<\infty
$$

Using again Lemma 4, statement 2, we conclude that

$$
A_{\omega} S_{1}(\cdot, f) \in L^{p}(0,1 ; X)
$$

if and only if

$$
Q_{\omega}^{2} e^{x Q_{\omega}} \Pi_{\omega}^{-1} d_{0} \in L^{p}(0,1 ; X)
$$

and thus

$$
\Pi_{\omega}^{-1} d_{0} \in\left(D\left(Q_{\omega}^{2}\right), X\right)_{\frac{1}{2 p}, p}=(D(A), X)_{\frac{1}{2 p}, p}
$$




\section{Applications}

In this section we give some applications for our abstract results.

Example 1. Let $X=L^{2}(] 0,1[)$. Consider operators $Q_{\omega}$ and $H$ in $X$, defined by

$$
\left\{\begin{array}{l}
D\left(Q_{\omega}\right)=D(H)=\left\{\varphi \in H^{2}(] 0,1[): \varphi(0)=\varphi(1)=0\right\} \\
\left(Q_{\omega} \varphi\right)(y)=\varphi^{\prime \prime}(y)+a(y) \varphi^{\prime}(y)-\sqrt{\omega} \varphi(y), y \in(0,1) \\
(H \varphi)(y)=-\varphi^{\prime \prime}(y), y \in(0,1)
\end{array}\right.
$$

Suppose that $a \in C^{2}([0,1])$ with $a(0)=a(1)=0$ and $\omega>0$ (large enough).

1. It is not difficult to prove that $Q_{\omega}, Q_{\omega}-H$ and $H$ are boundedly invertible.

Therefore

$$
\left\{\begin{array}{l}
D\left(Q_{\omega}-H\right)=\left\{\varphi \in H^{2}(] 0,1[): \varphi(0)=\varphi(1)=0\right\}, \\
\left(Q_{\omega}-H\right) \varphi(y)=2 \varphi^{\prime \prime}(y)+a(y) \varphi^{\prime}(y)-\sqrt{\omega} \varphi(y), \quad y \in(0,1)
\end{array}\right.
$$

(see for instance Cheggag et al. in [3]).

2. By using A. Lunardi [5, Theorem 3.1.3, p. 73], we obtain that the operator $Q_{\omega}=$ $-\left(-A_{\omega}\right)^{1 / 2}$ is well defined and generates an analytic semigroup. We also have

$$
D\left(A_{\omega}\right)=D\left(Q_{\omega}^{2}\right)=\left\{\varphi \in H^{4}(] 0,1[): \varphi^{\prime \prime}(0)=\varphi^{\prime \prime}(1)=\varphi(0)=\varphi(1)=0\right\},
$$

and

$$
\begin{gathered}
A_{\omega} \varphi(y)=-Q_{\omega}^{2} \varphi(y)=-\varphi^{(4)}(y)-2 a(y) \varphi^{(3)}(y)-\left(a^{2}(y)+2 a^{\prime}(y)-2 \sqrt{\omega}\right) \varphi^{\prime \prime}(y)- \\
-\left[a^{\prime \prime}(y)+a(y)\left(a^{\prime}(y)-2 \sqrt{\omega}\right)\right] \varphi^{\prime}(y)-\omega \varphi(y) .
\end{gathered}
$$

On the other hand, there exist conxtants $\delta, C>0$ such that for $z \in S_{\delta}=$ $\{z \in \mathbb{C} \backslash\{0\}:|\arg z| \leq \pi / 2+\delta\}$ we have

$$
\left\|\left(A_{\omega}-z I\right)^{-1}\right\|_{\left.L^{2}(] 0,1\right]} \leq \frac{C}{|\sqrt{\omega}+z|} .
$$

3. We obtain, for a.e. $y \in(0,1)$

$$
\left(H^{-1} \psi\right)(y)=(1-y) \int_{0}^{y} s \psi(s) d s+y \int_{y}^{1}(1-s) \psi(s) d s,
$$

so that

$$
\begin{gathered}
\left(Q_{\omega} H^{-1} \psi\right)(y)=Q_{\omega}\left(H^{-1} \psi\right)(y)= \\
=\left(H^{-1} \psi\right)^{\prime \prime}(y)+a(y)\left(H^{-1} \psi\right)^{\prime}(y)-\sqrt{\omega}\left(H^{-1} \psi\right)(y)
\end{gathered}
$$

then we get

$$
\begin{gathered}
\left(Q_{\omega} H^{-1} \psi\right)(y)=-\psi(y)-a(y) \int_{0}^{y} s \psi(s) d s+a(y) \int_{y}^{1}(1-s) \psi(s) d s- \\
-\sqrt{\omega}\left(\int_{0}^{y}(1-y) s \psi(s) d s+\int_{y}^{1} y(1-s) \psi(s) d s\right) .
\end{gathered}
$$


Indeed, we have

$$
\begin{gathered}
\left(H^{-1} Q_{\omega} \psi\right)(y)=H^{-1}\left(Q_{\omega} \psi\right)(y)= \\
=(1-y) \int_{0}^{y} s\left(Q_{\omega} \psi\right)(s) d s+y \int_{y}^{1}(1-s)\left(Q_{\omega} \psi\right)(s) d s= \\
=(1-y) \int_{0}^{y} s \psi^{\prime \prime}(s) d s+\int_{0}^{y} a(s) \psi^{\prime}(s) d s-\sqrt{\omega} \int_{0}^{y} \psi(s) d s+ \\
+y \int_{y}^{1}(1-s) \psi^{\prime \prime}(s) d s+\int_{y}^{1} a(s) \psi^{\prime}(s) d s-\sqrt{\omega} \int_{y}^{1} \psi(s) d s= \\
=-\psi(y)+(1-y) \int_{0}^{y} s a(s) \psi^{\prime}(s) d s+y \int_{y}^{1}(1-s) a(s) \psi^{\prime}(s) d s- \\
-\sqrt{\omega}\left((1-y) \int_{0}^{y} s \psi(s) d s+y \int_{y}^{1}(1-s) \psi(s) d s\right)
\end{gathered}
$$

and deduce that

$$
\forall \psi \in D\left(Q_{\omega}\right), Q_{\omega} H^{-1} \psi \neq H^{-1} Q_{\omega} \psi .
$$

Therefore, all our assumptions are satisfied. We have the following:

Proposition 1. Let $p \in] 1, \infty\left[, f \in L^{p}\left(0,1 ; L^{2}(] 0,1[)\right)\right.$ and

$$
\Pi_{\omega}^{-1} d_{0}, u_{1} \in\left(H^{4}(] 0,1[), L^{2}(] 0,1[)\right)_{\frac{1}{2 p}, p},
$$

where

$$
\Pi_{\omega}=Q_{\omega}-H+\left(Q_{\omega}+H\right) e^{2 Q_{\omega}} .
$$

Then, there exists $\omega^{*}>0$ such that for all $\omega \geq \omega^{*}$ the problem

$$
\left\{\begin{array}{l}
\frac{\partial^{2} u}{\partial x^{2}}(x, y)-\frac{\partial^{4} u}{\partial y^{4}}(x, y)-2 a(y) \frac{\partial^{3} u}{\partial y^{3}}(x, y)-\left(a^{2}(y)+2 a^{\prime}(y)-2 \sqrt{\omega}\right) \frac{\partial^{2} u}{\partial y^{2}}(x, y)- \\
-\left[a^{\prime \prime}(y)+a(y)\left(a^{\prime}(y)-2 \sqrt{\omega}\right)\right] \frac{\partial u}{\partial y}(x, y)-\omega u(x, y)=f(x, y), \quad x, y \in(0,1) \\
\frac{\partial u}{\partial x}(0, y)+\frac{\partial^{2} u}{\partial y^{2}}(0, y)=d_{0}(y), \quad y \in(0,1), \\
u(1, y)=u_{1}(y), \quad y \in(0,1), \\
u(x, 0)=u(x, 1)=0 \\
\frac{\partial^{2} u}{\partial y^{2}}(x, 0)=\frac{\partial^{2} u}{\partial y^{2}}(x, 1)=0
\end{array}\right.
$$

has a classical solution $u$, that is

$$
u \in W^{2, p}\left(0,1 ; L^{2}(] 0,1[)\right) \cap L^{p}\left(0,1 ; H^{4}(] 0,1[)\right), 1<p<\infty,
$$

and $u$ satisfies (41). 


\section{References}

1. Cheggag M., Favini A., Labbas R., Maingot S., Medeghri A. Sturm-Liouville Problems for an Abstract Differential Equation of Elliptic Type in UMD Spaces. Differential and Integral Equations, 2008, vol. 21, no. 9-10, pp. 981-1000.

2. Cheggag M., Favini A., Labbas R., Maingot S., Medeghri A. Abstract Differential Equations of Elliptic Type with General Robin Boundary Conditions in Hölder Spaces. Applicable Analysis: an International Journal, 2012, vol. 91, no. 8, pp. 1453-1475.

3. Cheggag M., Favini A., Labbas R., Maingot S., Ould Melha K. New Results on Complete Elliptic Equations with Robin Boundary Coefficient-Operator Conditions in Non Commutative Case. Bulletin of the South Ural State University. Series: Mathematical Modelling, Programming and Computer Software, 2017, vol. 10, no. 1, pp. 70-96. DOI: $10.14529 / \mathrm{mmp} 170105$

4. Dore G., Yakubov S. Semigroup Estimates and Noncoercive Boundady Value Problems. Semigroups Forum, 2000, no. 60, pp. 93-121.

5. Lunardi A. Analytic Semigroups and Optimal Regularity in Parabolic Problems. Basel, Birkhaüser, 1995.

6. Grisvard P. Spazi di tracce ed applicazioni. Rendiconti di Matematica. Serie VI, 1972, vol. 5, pp. 657-729. (in Italian)

7. Cheggag M., Favini A., Labbas R., Maingot S., Medeghri A. Elliptic Problems with Robin Boundary Coefficient-Operator Conditions in General $L_{p}$ Sobolev Spaces and Applications. Bulletin of the South Ural State University. Series: Mathematical Modelling, Programming and Computer Software, 2015, vol. 8, no. 3, pp. 56-77. DOI: 10.14529/mmp150304

8. Prüss J., Sohr H. On Operators with Bounded Imaginary Powers in Banach Spaces. Mathematische zeitschrift, 1990, vol. 203, no. 3, pp. 429-452.

9. Grisvard P. Commutativité de deux foncteurs d'interpolation et applications. Journal de mathématiques pures et appliquées, 1966, no. 45, pp. 143-290.

10. Triebel H. Interpolation Theory, Functions Spaces, Differential Operators. Amsterdam, N.Y., Oxford, North-Holland, 1978.

11. Favini A., Labbas R., Maingot S., Tanabe H., Yagi A. A Simplified Approach in the Study of Elliptic Differential Equation in UMD Space and New Applications. Funkcialaj ekvacioj, 2008, vol. 51, no. 2, pp. 165-187.

12. Dore G., Venni A. On the Closedness of the Sum of Tow Closed Operators. Mathematische zeitschrift, 1987, vol. 196, no. 2, pp. 189-201. DOI: 10.1007/BF01163654

13. Favini A., Labbas R., Maingot S., Tanabe H., Yagi A. Complete Abstract Differential Equation of Elliptic Type in U.M.D Spaces. Funkcialaj ekvacioj, 2006, vol. 49, no. 2, pp. 193-214.

Received June 3, 2018 


\title{
АБСТРАКТНЫЕ ЗАДАЧИ ШТУРМА-ЛИУВИЛЛЯ ДЛЯ ДИФФЕРЕНЦИАЛЬНЫХ УРАВНЕНИЙ ВТОРОГО ПОРЯДКА В НЕКОММУТАТИВНОМ СЛУЧАЕ
}

\author{
Хенд Магомет ${ }^{1}$, Хеллаф Улъд Мелха \\ ${ }^{1}$ Университет имени Абдул Хамид Ибн-Бадиса, г. Мостаганем, Алжир
}

\begin{abstract}
В статье доказываются некоторые новые результаты о задаче Штурма - Лиувилля для дифференциальных уравнений эллиптического типа второго порядка в некоммутативном случае. Исследование выполнено при условии, что второй член принадлежит пространству Соболева. Доказано существование, единственность и оптимальная регулярность строгого решения. Работа является продолжением исследований в коммутативном случае М. Чегага, А. Фавини, Р. Лаббаса, С. Менго и А. Медегри. В работе рассматривается пример приложения построенной абстрактной теории.

Ключевые слова: эллиптическое дифберенциальное уравнение второго порядка; задача ШІтурма - Лиувилля в некоммутативном случае; аналитическая полугруппа; максимальная регулярность.
\end{abstract}

Хенд Магомет, лаборатория чистой и прикладной математики, Университет имени Абдул Хамид Ибн-Бадиса (г. Мостаганем, Алжир), mohammed.kaid@univ-mosta.dz.

Хеллаф Ульд Мелха, лаборатория чистой и прикладной математики, Университет имени Абдул Хамид Ибн-Бадиса (г. Мостаганем, Алжир), ould_melha_khel@yahoo.fr.

Поступила в редакиию 3 июня 2018 г. 